Elect. Comm. in Probab. 16 (2011), 623-629

\title{
A MULTIPLICATIVE SHORT PROOF FOR THE UNIMODALITY OF STABLE DENSITIES
}

\author{
THOMAS SIMON \\ Laboratoire Paul Painlevé, Université Lille 1, 59655 Villeneuve d’Ascq Cedex, France. \\ email: simon@math.univ-lille1.fr
}

Submitted January 6, 2011, accepted in final form October 9, 2011

AMS 2000 Subject classification: 60E07, 60E15

Keywords: Stable law, unimodality

\begin{abstract}
Revisiting an article by Chernin and Ibragimov on unimodality of stable laws, we show that their approach to deduce the general case from the extremal ones, whose completion contained an error as discovered later by Kanter, can be carried out successfully in considering Bochner's subordination and multiplicative strong unimodality. This short proof of the unimodality of all stable densities yields also a multiplicative counterpart to Yamazato's additive ones.
\end{abstract}

\section{Introduction}

A real random variable $X$ is said to be unimodal if there exists $a \in \mathbb{R}$ such that its distribution function $\mathbb{P}[X \leq x]$ is convex on $(-\infty, a)$ and concave on $(a,+\infty)$. The number $a$ is called a mode of $X$, and might not be unique. Unimodality is a basic property for real random variables, with some applications in deviation inequalities and statistics, and we refer to the monograph [3] for more on this subject. Concluding a series of attempts by several authors, the following was finally obtained in [17]:

Theorem (Yamazato). All stable laws are unimodal.

The original argument in [17] is very robust, extending to all self-decomposable distributions, and also quite tricky. A simpler proof relying on Stieltjes transforms and additive strong unimodality, had then been proposed in [18] pp. 600-601. The purpose of the present note is to provide yet another short proof of the unimodality for all stable laws hinging on Bochner's subordination and multiplicative strong unimodality in the sense of [2]. We will also somehow fulfil the original project of Chernin and Ibragimov who had wished in [1] to deduce the general property from the simpler spectrally one-sided and symmetric cases. It is well-known [10] that their approach to fill the gap between these particular stable laws and general ones, based on harmonic functions, turned out to contain an error which is not retrievable. In [19] p. 122, remembering things past and with a view towards [1], Zolotarev regrets that "there is not yet an independent analytic proof of unimodality for stable laws". 
In this paper we aim at completing the results of [1] in an analytical way, with a different method. Cutting off stable densities at zero, we first easily observe that one of the two resulting densities is monotone. For the second, non-monotone one, Bochner's subordination allows first to reexpress the problem in terms of a power transformation of the quotient of two positive stable random variables. From Kanter's factorization [9] which is a direct consequence of the final remark in [1], we then have to consider the independent product of four random variables. The first two are power transformations of the exponential law and immediately shown to be multiplicatively strong unimodal, whereas the last two are convex resp. concave monotonous transformations of the uniform law, whose product is easily proved to be unimodal.

Both Yamazato's proofs in $[17,18]$ used strong unimodality in the additive framework. We feel that multiplicative strong unimodality which is a less classical topic, is also a cosy tool. With its help we can also show very quickly (except in the skewed Cauchy case) that stable laws are strictly unimodal viz. their mode is unique - see Remark (b) below. Notice that multiplicative strong unimodality allows to handle spectrally one-sided stable variables as well - see Remark (d) below, which shows overall that with this method the general case can be deduced from the final remark in [1] only.

\section{Proof of the theorem}

We begin with a fairly obvious result which is reminiscent of Lemma 52.10 in [12], and which will play the same rôle in our argument as the latter did in $[17,18]$.

Lemma. Let $F:(0,1) \rightarrow\left(0, c_{F}\right)$ be increasing concave and $G:(0,1) \rightarrow\left(c_{G},+\infty\right)$ be increasing convex, with $c_{F}$ and $c_{G}$ positive and finite. Let $U, V$ be two independent copies of the uniform law on $(0,1)$. The random variable

$$
T=F(U) \times G(V)
$$

is unimodal with mode at $c_{F} c_{G}$.

Proof. After changing the variable, it is easy to see that one just needs to consider the case $c_{F}=$ $c_{G}=1$. Set $X=F(U)$ and $Y=G(V)$. Since $F$ is increasing concave, $X$ is absolutely continuous with non-decreasing density $f_{X}$ on $(0,1)$. Similarly, since $G$ is increasing convex, $Y$ is absolutely continuous with non-increasing density $f_{Y}$ on $(1,+\infty)$. If $x \leq 1$, one has

$$
f_{T}(x)=\int_{0}^{\infty} f_{Y}(x / u) f_{X}(u) \frac{d u}{u}=\int_{0}^{x} f_{Y}(x / u) f_{X}(u) \frac{d u}{u}=\int_{0}^{1} f_{Y}(1 / v) f_{X}(x v) \frac{d v}{v}
$$

which is non-decreasing. If $x \geq 1$, one has

$$
f_{T}(x)=\int_{0}^{\infty} f_{X}(x / u) f_{Y}(u) \frac{d u}{u}=\int_{x}^{\infty} f_{X}(x / u) f_{Y}(u) \frac{d u}{u}=\int_{1}^{\infty} f_{X}(1 / v) f_{Y}(x v) \frac{d v}{v}
$$

which is non-increasing.

We now proceed to the proof of Yamazato's theorem. We first deal with strictly stable laws and we will use Zolotarev's third parametrization as explained in [19] p. 17. Let $X(\alpha, \theta, \lambda)$ be strictly stable associated with the three parameters $(\alpha, \theta, \lambda)$. Notice first that since $-X(\alpha, \theta, \lambda)$ has the 
same law as $X(\alpha,-\theta, \lambda)$, it is enough to consider the case where $\theta \geq 0$ i.e. the positivity parameter $\rho=(1+\theta) / 2$ belongs to $[1 / 2,1]$. Also, without loss of generality one can choose $\lambda=1$, and we will use the notation $X(\alpha, \theta, 1)=X(\alpha, \rho)$.

Consider first the case $\rho=1$. Notice that it follows from Lemma 1 in [1], but we will give here a different proof in order to introduce our method. Recall that necessarily $\alpha \leq 1$ and $X(\alpha, 1) \geq 0$ a.s. The Laplace transform is given by

$$
\mathbb{E}\left[e^{-t X(\alpha, 1)}\right]=e^{-t^{\alpha}}, \quad t \geq 0,
$$

and henceforth we will set $X(\alpha, 1)=T_{\alpha}$ for simplicity. One has $T_{1}=1$ a.s. and constant random variables are unimodal. When $\alpha<1$, the contour integration mentioned in [1] - see [8] pp. 68-69 for details on the "necessary computations" - yields the so-called Kanter's factorization as described in Corollary 4.1 of [9]:

$$
T_{\alpha} \stackrel{d}{=} L^{(\alpha-1) / \alpha} \times b_{\alpha}^{-1 / \alpha}(U)
$$

where $L \sim \operatorname{Exp}(1), U \sim \operatorname{Unif}(0,1)$ independent of $L$, and

$$
b_{\alpha}(u)=(\sin \pi u / \sin (\alpha \pi u))^{\alpha}(\sin \pi u / \sin ((1-\alpha) \pi u))^{1-\alpha}, \quad u \in(0,1) .
$$

We had proved in Lemma 1 of [15] that $b_{\alpha}$ is decreasing concave on $(0,1)$, so that the function $u \mapsto b_{\alpha}^{-1 / \alpha}(u)$ is increasing convex on $(0,1)$ and the variable $b_{\alpha}^{-1 / \alpha}(U)$ is unimodal (with nonincreasing density). On the other hand, the variable $\log L$ is easily seen to have a $\log$-concave density on $\mathbb{R}$ so that $L^{(\alpha-1) / \alpha}$ is multiplicatively strong unimodal (MSU) in the sense of [2] - see Theorem 3.7 therein. This shows that $T_{\alpha}$ is unimodal.

Next, consider the case $1 / 2 \leq \rho<1$ and set $X_{\rho}=X(1 / \rho, \rho)$ for the associated completely asymmetric stable random variable. Bochner's subordination for Lévy processes - see e.g. Chapter 6 in [12] for details - and self-similarity entail readily that $X(\alpha, \rho)$ can be viewed as the independent product

$$
X(\alpha, \rho) \stackrel{d}{=} T_{\alpha \rho}^{\rho} \times X_{\rho}
$$

The density $f_{\alpha, \rho}$ of $X(\alpha, \rho)$ is hence given by

$$
f_{\alpha, \rho}(x)=\int_{0}^{\infty} f_{X_{\rho}}(x / u) f_{T_{\alpha \rho}^{\rho}}(u) \frac{d u}{u}
$$

for all $x \in \mathbb{R}$ with obvious notations for $f_{X_{\rho}}$ and $f_{T_{\alpha \rho}}$, and since we know from Lemma 1' in [1] - beware Zolotarev's second parametrization $(\alpha, \beta, \lambda)$ therein, and see Remark (d) below for an independent proof - that $f_{X_{\rho}}$ is increasing on $\mathbb{R}^{-}$, the same holds for $f_{\alpha, \rho}$. All in all, recalling that $f_{\alpha, \rho}$ is continuous at zero, it is enough to show that the cut off random variable

$$
X^{+}(\alpha, \rho)=X(\alpha, \rho) \mid X(\alpha, \rho) \geq 0
$$

is unimodal. When $\rho=1 / 2$, our normalization yields $X_{1 / 2}=\sqrt{2} \mathscr{N}$ where $\mathscr{N}$ is standard normal, so that $f_{X_{1 / 2}}$ is decreasing on $\mathbb{R}^{+}$and one can reason as above. When $\rho>1 / 2$, we first notice that

$$
X^{+}(\alpha, \rho) \stackrel{d}{=}\left(T_{\alpha \rho}^{\rho} \times X_{\rho}\right) \mid X_{\rho} \geq 0 \stackrel{d}{=} \kappa_{\rho}\left(\frac{T_{\alpha \rho}}{T_{\rho}}\right)^{\rho}
$$


for some irrelevant, positive finite constant $\kappa_{\rho}=(2|\cos (\pi / 2 \rho)| / \pi)^{-\rho}$, where the second identity in law is a well-known, particular case of the so-called Zolotarev duality - see Section 2.3 in [19] - which can be obtained e.g. in solving Exercises 29.7 and 29.18 in [12]. To conclude, we first deduce from (2.1) the factorization

$$
\left(\frac{T_{\alpha \rho}}{T_{\rho}}\right)^{\rho} \stackrel{d}{=} L^{(\alpha \rho-1) / \alpha} \times L^{1-\rho} \times b_{\alpha \rho}^{-1 / \alpha}(U) \times b_{\rho}(1-U),
$$

where all four random variables on the right-hand side are independent. On the one hand, the density of $\log \left(L^{(\alpha \rho-1) / \alpha} \times L^{1-\rho}\right)$ is log-concave by Prékopa's theorem - see e.g. the beginning Section 1.1 in [11] for the latter - so that $L^{(\alpha \rho-1) / \alpha} \times L^{1-\rho}$ is MSU. On the other hand, $u \mapsto b_{\alpha \rho}^{-1 / \alpha}(u)$ is increasing convex from $[0,1)$ to $\left[c_{\alpha, \rho},+\infty\right)$ (with $\left.c_{\alpha, \rho}=(\alpha \rho)^{\rho / \alpha}(1-\alpha \rho)^{(1 / \alpha-\rho)}\right)$ and $u \mapsto b_{\rho}(1-u)$ is increasing concave from $[0,1]$ to $\left[0, c_{\rho}\right]$ (with $c_{\rho}=\rho^{-\rho}(1-\rho)^{\rho-1}$ ), so that $b_{\alpha \rho}^{-1 / \alpha}(U) \times b_{\rho}(1-U)$ is unimodal by the Lemma. This completes the proof in the strictly stable case.

We finally consider general stable laws. When the self-similarity index $\alpha$ is not 1 , they are obtained from the strictly $\alpha$-stable ones by a linear shift - see e.g. Theorem 14.8 (i) in [12], which does not affect unimodality. When $\alpha=1$, the situation is more complicated and non strictly 1-stable laws cannot be recovered from strictly 1-stable ones in such a direct way - see Theorem 14.8 (ii) in [12]. However, up to some explicit increasing change of variable which is described in [19] pp. 72-73, non strictly 1-stable laws are weak limits of $\alpha$-stable ones with $\alpha \neq 1$. Hence it is enough to check that unimodality remains unchanged under weak convergence, a fact which was first proved by Lapin - see Theorem 4 p. 160 in [6] or Exercise 29.20 in [12].

Remarks. (a) The short proof which is sketched in the final remark of [18] used a decomposition of $X(\alpha, \rho)$ as the sum of four random variables, the first two being convergent sums of translated exponential random variables, hence additively strongly unimodal by Lemma 52.4 in [12], whereas the last two have monotone densities in opposite half-lines, so that their sum is unimodal by Lemma 52.10 in [12]. The reason why the latter random variables have monotone densities comes from Steutel's characterization of Stieltjes transforms of one-sided probabilities see Lemma 4.1. in [18] - and a suitable discretization of the Lévy-Khintchine formula somehow reminiscent of the one in [17]. The number four and the general notion of strong unimodality are hence common to our proof and Yamazato's in [18], but it seems impossible to deduce one from the other, and both remain esssentially different.

(b) It is easily seen from the proof of Lemma 1 in [15] that the smooth function $b_{\gamma}$ is strictly concave for every $0<\gamma<1$, hence its negative power transformations are strictly convex. This entails from the proof of the Lemma in the present paper that the density of $b_{\alpha \rho}^{-1 / \alpha}(U) \times b_{\rho}(1-U)$ has positive derivative on $\left(0, c_{\rho} c_{\alpha, \rho}\right)$ and negative derivative on $\left(c_{\rho} c_{\alpha, \rho},+\infty\right)$, and a perusal of Step 6 p. 212 in [2] shows then without difficulty that $X^{+}(\alpha, \rho)$ is strictly unimodal for $1 / 2<\rho<1$ (with a positive mode since

$$
f_{\alpha, \rho}^{\prime}(0)=f_{X_{\rho}}^{\prime}(0) \int_{0}^{\infty} f_{T_{\alpha \rho}^{\rho}}(u) \frac{d u}{u^{2}}>0,
$$

the strict inequality coming from $f_{X_{\rho}}^{\prime}(0)>0$ - see Formula (14.30) in [12]). This proves that all $X(\alpha, \rho)$ are strictly unimodal except perhaps for $\rho=0,1 / 2$ or 1 . The case $\rho=1 / 2$ is a 
straightforward consequence of (2.2) and the Gaussian explicit density - see Remark (e) below, and the one-sided cases $\rho=0$ or 1 can be handled exactly as above. This proves strict unimodality of all strictly stable laws, and to get it for the non strictly ones requires no further effort except for $\alpha=1$. Unfortunately, since strict unimodality might not be preserved under weak convergence, in the discussion at the end of the above proof we cannot conclude so quickly that skewed Cauchy laws are strictly unimodal. Using Zolotarev's second parametrization, Theorem 2.2.3. in [19] and a change of variables yields also the following Kanter type factorization for skewed Cauchy random variables:

$$
X(1, \beta) \stackrel{d}{=} \beta\left(\log \left(U_{1}(\Phi, \beta)\right)-\log L\right)
$$

with $\Phi$ uniform on $(-1,1), L \sim \operatorname{Exp}(1)$ and an explicit function $U_{1}(\varphi, \beta)$. The variable $\log L$ is additively strong unimodal but since the increasing function $\log U_{1}(\varphi, \beta)$ is clearly neither concave nor convex in $\varphi$, proving the unimodality or strict unimodality of $\log \left(U_{1}(\Phi, \beta)\right)$ seems not easy, so that we must refer to the more general Theorem 1.4 in [13] for a proof of the strict unimodality of $X(1, \beta)$.

(c) By the Lemma, the right-hand side of (2.3) yields a product of three independent random variables whose unique modes are completely explicit. Notice that this is not so in [18] where the two additively strongly unimodal random variables are obtained as weak limits. However, this more precise feature does not seem to give any valuable information on the exact location of the mode of $X^{+}(\alpha, \rho)$ viz. of $X(\alpha, \rho)$, because modes change usually quite fuzzily after independent multiplication. We refer to Theorem 2.1. in [7] for a limit theorem on the location of the mode of stable random variables when $\beta \rightarrow 0$ with Zolotarev's second parametrization i.e. $\rho \rightarrow 1 / 2$ in the strictly stable case.

(d) The crucial fact that $f_{X_{\rho}}$ is increasing on $\mathbb{R}^{-}$depends on Lemma 1' of [1]. We stress that it is also possible to derive this property from Bochner's subordination, the MSU property and Zolotarev's duality. Even though this argument is longer, we will give the details since overall it shows that in [1], the sole final remark would have been enough to derive everything. Recall from formula (14.30) in [12] that $f_{X_{\rho}}^{\prime}(0)>0$, so that we only need to prove that $X_{\rho}$ restricted on $\mathbb{R}^{-}$ is unimodal. Using the notation $X^{+}=X \mid X>0$ for any real random variable $X$, this amounts to show that

$$
X^{+}(1 / \rho, 1-\rho) \stackrel{d}{=} X^{+}(\rho, 1 / \rho-1)^{-\rho}
$$

is unimodal, the identity in law following from Zolotarev's duality - see (2.3.3) in [19]. Setting $\rho^{\prime}=1 / \rho-1$, suppose first that $\rho^{\prime} \leq 1 / 2$ i.e. $\rho \geq 2 / 3$. One has

$$
X^{+}\left(\rho, \rho^{\prime}\right)^{-\rho} \stackrel{d}{=}\left(T_{\rho} \times X^{+}\left(1, \rho^{\prime}\right)\right)^{-\rho} \stackrel{d}{=} T_{\rho}^{-\rho} \times\left(\frac{T_{\rho^{\prime}}}{T_{\rho}^{\prime}}\right)^{\rho \rho^{\prime}}
$$

where the first identity follows from Bochner's subordination and the well-known second one e.g. in inverting the Mellin transform given in [19], Theorem 2.6.3. On the one hand, the unimodality of $T_{\rho}^{-\rho}$ follows from Kanter's factorization and the concavity of $b_{\rho}$, as mentioned above. On the other hand, the independent quotient on the right-hand side is MSU by the main result of [14] because $\rho^{\prime} \leq 1 / 2$, so that we are done. Last, suppose $\rho^{\prime}>1 / 2$ i.e. $1 / 2<\rho<2 / 3$. Again, Bochner's subordination and Zolotarev's duality entail

$$
X^{+}\left(\rho, \rho^{\prime}\right)^{-\rho} \stackrel{d}{=} T_{\rho \rho^{\prime}}^{-\rho \rho^{\prime}} \times X^{+}\left(1 / \rho^{\prime}, \rho^{\prime}\right)^{-\rho} \stackrel{d}{=}\left(\frac{T_{\rho^{\prime}}}{T_{\rho \rho^{\prime}}}\right)^{\rho \rho^{\prime}} .
$$


The variable $T_{\rho^{\prime}}^{\rho \rho^{\prime}}$ is unimodal from Kanter's factorization and the convexity of $b_{\rho^{\prime}}^{-\rho}$ - see also [15] for a more general result, whereas the variable $T_{\rho \rho^{\prime}}^{-\rho \rho^{\prime}}$ is MSU by the main result of [14], because $\rho \rho^{\prime}=1-\rho<1 / 2$. This completes the argument.

(e) When $\rho=1 / 2$, the formula (2.2) reads

$$
f_{\alpha, 1 / 2}(x)=\frac{1}{2 \sqrt{\pi}} \int_{0}^{\infty} e^{-x^{2} / 4 u^{2}} f_{T_{\alpha / 2}^{1 / 2}}(u) \frac{d u}{u}=\frac{1}{\sqrt{\pi}} \int_{0}^{\infty} e^{-x^{2} / 4 u^{2}} f_{T_{\alpha / 2}}\left(u^{2}\right) d u
$$

which can also be derived in inverting directly the Fourier transform $e^{-|\lambda|^{\alpha}}$ - see Section 11 in [16] and beware the minor misprint in (34) therein. This original way to prove the unimodality of symmetric stable laws remains the quickest of all.

(f) If $X$ is a real random variable with smooth density, the so-called bell-shape property means that the $n$-th derivative of this density vanishes exactly $n$ times. This property which is much stronger than unimodality, is true for Gaussian densities because of the underlying Hermite polynomials, and also for the explicit Cauchy and Lévy densities by a direct analysis. It is claimed in [4] for general stable laws, but the proof contains a serious error because of the false assertion made in Lemma 1 therein that the density of the logarithm of the positive stable random variable yields always a strictly totally positive kernel - see Remark (a) in [14] for details. This error does not seem amendable in a direct way [5], and one might wonder if the methods of the present note could not be helpful to tackle this difficult question.

Acknowledgements. Part of this paper was written during a stay at the University of Tunis. I wish to thank W. S. Jedidi for his kind hospitality and grants ANR-09-BLAN-0084-01 and CMCU10 G1503 for support. I am also grateful to M. Yamazato for having drawn my attention to [18].

\section{References}

[1] K. E. Chernin and I. A. Ibragimov. On the unimodality of stable laws. Teor. Veroyatnost. $i$ Primenen. 4, 453-456, 1959. MR0116382

[2] I. Cuculescu and R. Theodorescu. Multiplicative strong unimodality. Austral. \& New Zealand J. Statist. 40 (2), 205-214, 1998. MR1631092

[3] S. Dharmadhikari and K. Joag-Dev. Unimodality, convexity and applications. Academic Press, New-York, 1988. MR0954608

[4] W. Gawronski. On the bell-shape of stable densities. Ann. Probab. 12 (1), 230-242, 1984. MR0723742

[5] W. Gawronski. Personal communication to W. S. Jedidi, 2001.

[6] B. V. Gnedenko and A. N. Kolmogorov. Limit distributions for sums of independent random variables. Revised edition. Addison-Wesley, Reading, 1968. MR0233400

[7] P. Hall. On unimodality and rates of convergence for stable laws. J. London Math. Soc. 30 (2), 371-384, 1984. MR0771431 
[8] I. A. Ibragimov and Yu. V. Linnik. Independent and stationary sequences of random variables. Wolters-Noordhoff, Groningen, 1971. MR0322926

[9] M. Kanter. Stable densities under change of scale and total variation inequalities. Ann. Probab. 3, 697-707, 1975. MR0436265

[10] M. Kanter. On the unimodality of stable densities. Ann. Probab. 4 (6), 1006-1008, 1976. MR0433544

[11] B. Maurey. Inégalité de Brunn-Minkowski-Lusternik, et autres inégalités géométriques et fonctionnelles. Astérisque 299, 95-113, 2005. MR2167203

[12] K. Sato. Lévy processes and infinitely divisible distributions. Cambridge University Press, Cambridge, 1999. MR1739520

[13] K. Sato and M. Yamazato. On distribution functions of class L. Z. Wahrsch. verw. Geb. 43, 273-308, 1978. MR0494405

[14] T. Simon. Multiplicative strong unimodality for positive stable laws. Proc. Amer. Math. Soc. 139 (7), 2587-2595, 2011. MR2784828

[15] T. Simon. On the unimodality of power transformations of positive stable densities. To appear in Mathematische Nachrichten.

[16] A. Wintner. Cauchy's stable distributions and an "explicit formula" of Mellin. Amer. J. Math. 78 (4), 819-861, 1956. MR0082217

[17] M. Yamazato. Unimodality of infinitely divisible distribution functions of class L. Ann. Probab. 6 (4), 523-531, 1978. MR0482941

[18] M. Yamazato. On strongly unimodal infinitely divisible distributions. Ann. Probab. 10 (3), 589-601, 1982. MR0659530

[19] V. M. Zolotarev. One-dimensional stable distributions. AMS, Providence, 1986. MR0854867 